\title{
Complex oxide nanomembranes for energy conversion and storage: A review- ERRATUM
}

Kian Kerman and Shriram Ramanathan

doi: 10.1557/jmr.2013.301, Published by Materials Research Society with Cambridge University Press, 4 November 2013.

In Kerman et al. ${ }^{1}$, page 1 , second column: $\mathrm{k}$ is the universal gas constant should read $\mathrm{k}$ is Boltzmann's constant.

The publisher regrets the mistake.

\section{REFERENCE}

1. K. Kerman and S. Ramanathan: Complex oxide nanomembranes for energy conversion and storage: A review. J. Mater. Res. (2013). 